\title{
Reconstruction enhancement via projection screening in holographic tomography
}

\author{
Piotr Machnio, ${ }^{*}$ Michał Ziemczonok, and Małgorzata Kujawińska \\ Warsaw University of Technology, Institute of Micromechanics and Photonics, \\ Św. A. Boboli 8 street, 02-525 Warsaw, Poland
}

Received May 27, 2021; accepted June 29, 2021; published June 30, 2021

\begin{abstract}
This paper presents an algorithm for automatic detection of erroneous amplitude and phase components of a sample's optical field, acquired by a holographic tomograph with a limited angle of projection. By applying image processing methods and statistical analysis to find and remove unfit projections, the quality of tomographic reconstruction of a 3D refractive index distribution of an object is greatly improved. The proposed methods can find their application in preprocessing of data in holographic tomography.
\end{abstract}

Limited projection angle holographic tomography (LAHT) is the most popular tool for 3D quantitative phase imaging of cells and tissues [1-2]. LAHT provides a $3 \mathrm{D}$ distribution of the refractive index (RI) and its changes in biological microstructures [3]. The measurement process consists of acquisition of digital holograms of a sample with different illumination directions, calculating the amplitude and phase maps of captured projections followed by numerical reconstruction of the 3D RI distribution (Fig. 1).

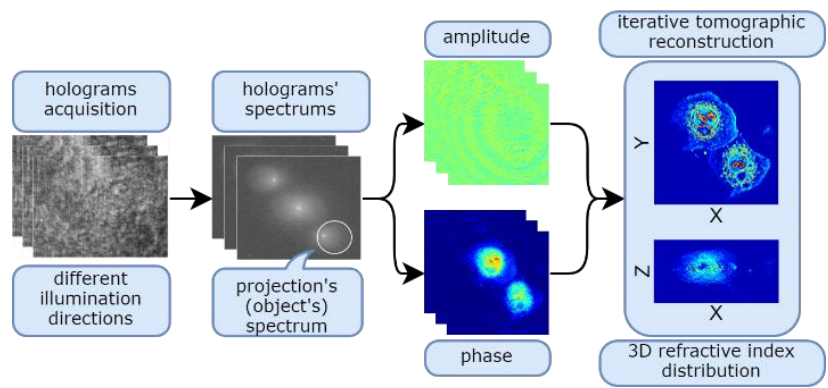

Fig. 1. Processing path in LAHT.

LAHT configuration is well-suited for biomedical applications, however, it suffers from drawbacks of the limited angle of projection, which causes degradation of quality and accuracy of measurement along the optical axis due to the missing cone problem [1] in the object's $3 \mathrm{D}$ spatial frequency spectrum. This problem is partially solved by means of iterative regularization [4]. However this reconstruction approach is strongly dependent on the quality of input (error free) projections [5]. For this reason, and because of sheer volume of data gathered

*E-mail: piotr.machnio.stud@pw.edu.pl during measurement, automatic methods for error detection must be developed.

Erroneous projections carry a substantial amount of information not associated with the object under study. They usually arise from low contrast of interferometric fringes caused by vibrations, dust particles or parasitic reflections, to name a few. The presence of these errors can, depending on their severity, degrade reconstruction and usually limits the effectiveness of iterative RI reconstruction procedures. Therefore, heavily damaged projections need to be removed to maximize the quality of reconstruction. To decide which projections should be removed we propose evaluating acquired data using three separate metrics.
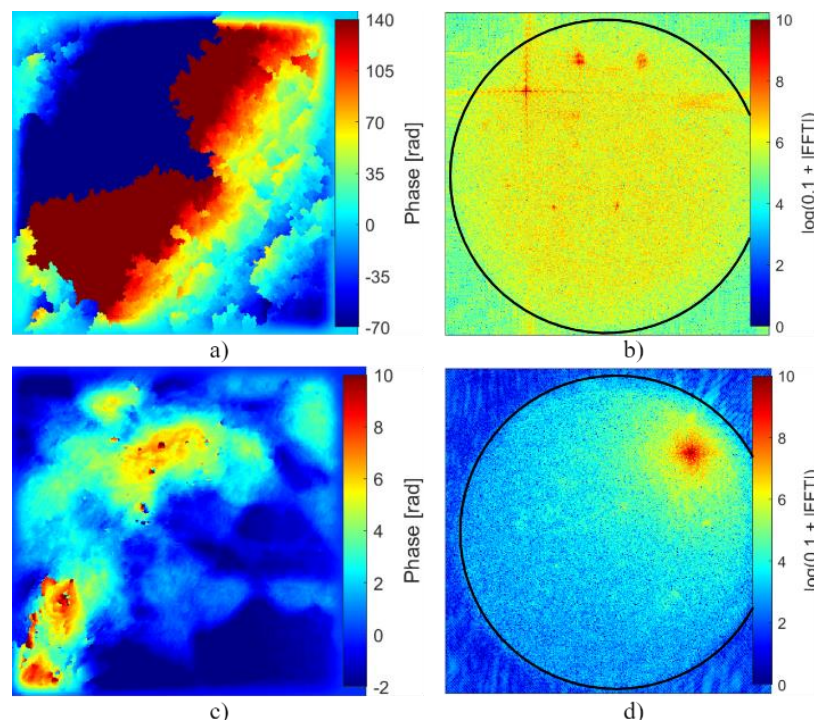

Fig. 2. Examples of projections: a), c) highly and weakly erroneous phase maps, b), d) their spectra with indicated aperture area.

The first proposed metric for detecting erroneous projections is based on the analysis of energy distribution within the projection's frequency spectrum (Fig. 2). Holographic tomograph systems rely on clear spectrum representation of the sample, which requires very limited noise in the frequency spectrum of the recorded digital holograms. Because of that, the frequency range allowed by the aperture of the optical system can be easily 
distinguished from the background, unless the projection is damaged (Fig. 2 b,d). Therefore, by comparing the average energy level outside of the aperture area, to the average energy level within the aperture area in projection's frequency spectrum, and then statistically analyzing the calculated energy ratios of all acquired projections, we can estimate which projections are very erroneous. The said energy ratio $S$ is calculated according to:

$$
S=\frac{\bar{E}_{\text {out }}}{\bar{E}_{\text {in }}-\bar{E}_{\text {out }}}
$$

where $\bar{E}_{\text {in }}, \bar{E}_{\text {out }}$ denote average energy inside and outside of the aperture, respectively. In order to assess whether projection needs to be removed we find the median and the standard deviation of $S$ within acquired dataset and remove projections with the $S$ values exceeding the threshold. The threshold calculation method is described by the pseudocode:

$$
\begin{gathered}
\text { threshold }=\min \left(\text { median }+k^{*}(\text { median }-\min (S))\right. \\
\text { median } \left.+k^{*} \text { standard deviation }\right) \\
\text { threshold }=\min (\max (\text { threshold }, \text { min limit }), \text { max limit })
\end{gathered}
$$

As a base for threshold, we use either the difference between median and minimum obtained ratio or standard deviation of ratios, whichever produces the lower threshold. This two-way approach proved to work properly on various measurements with ranging quantities of erroneous projections. Factor $k$ controls the sensitivity of detection (when $k$ grows sensitivity declines) and can be changed by a user. In our case, $k=2$ proved to be sufficient. Additionally, we introduced minimum limit and maximum limit of threshold, which are also controlled by the user. The minimum limit ensures that we do not remove projections which are still good but happened to be in dataset with projections of mainly pristine quality. On the other hand, maximum limit is introduced in a case where the majority of projections is erroneous, and the threshold calculated on their basis is too high. In the case of our system, the values of 0.5 and 1.2 for minimum limit and maximum limit respectively were optimal. The abovedescribed metric alone provides very good results, as it reliably detects completely destroyed projections which are the most harmful to the reconstruction. However, weakly erroneous phase projections, like the one presented in Fig. 2c, do not have a noisy spectrum (Fig. 2d) and can be not identified. Thus, we propose two additional methods which successfully complement the abovementioned algorithm.

The second method is based on the analysis of present high phase gradients in the projection. We use Prewitt's method of detecting gradients in $x$ and $y$ directions, and then calculate total gradient magnitude [6]. The phase unwrapping algorithm allows for jumps of phase of up to $|\pi|$ radians per pixel. Using that knowledge we can specify a maximum allowable gradient, and then calculate the sum of gradients exceeding the said limit within every projection. The obtained sum is expressed as relative to the total pixel count of the projection to compensate for various resolution. The resulting value is compared to the user specified threshold. Projections with higher gradients sum than the threshold are removed. In our case the threshold of 0.015 was optimal. The described method allowed us to detect projections with local phase errors, like those presented in Fig. 2c.

The final metric for detecting erroneous projections is based on searching for amplitude distributions which are significantly different than average in the given dataset. We noticed that extreme values of amplitude often coincide with erroneous phase (Fig. 3).
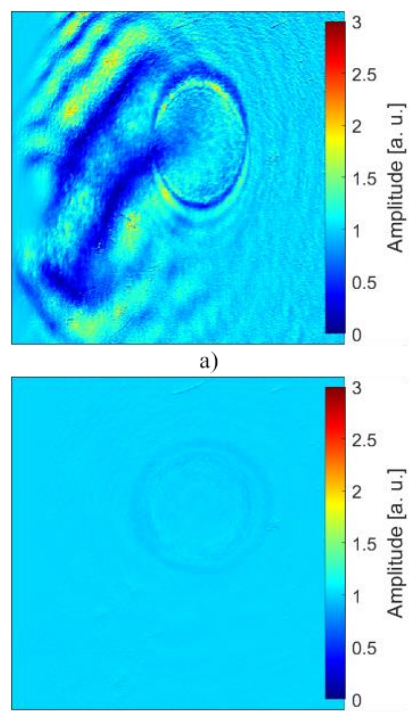

c)

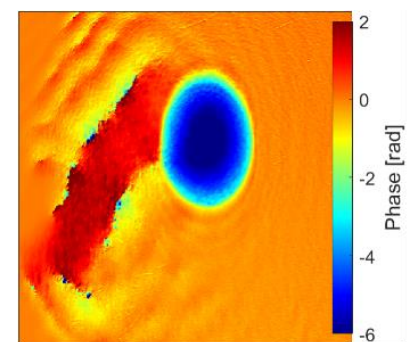

b)

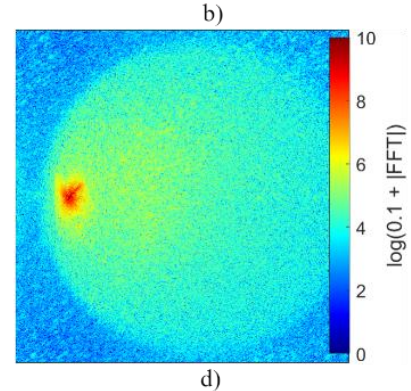

Fig. 3. Projection detected with amplitude metric: a) amplitude,

b) corresponding phase, c) median amplitude and d) spectrum.

To quantify the difference between each projection and average projection, we first calculate the median amplitude projection and then use it as a reference for calculating RMS differences. This approach for quantifying differences has limited reliability due to the fact that, depending on the measured object, some amplitude distributions vary significantly as the illumination direction changes, which in turn results in high fluctuations of obtained RMS differences. To overcome this problem, we include the range of RMS fluctuations in threshold calculations. The formula for obtaining threshold is as follows:

$$
T=Q_{75}+m *\left(Q_{75}-Q_{25}\right)
$$

where $Q_{25}$ and $Q_{75}$ are the 25-th and 75-th percentile of RMS differences respectively, and $m$ is the user specified parameter for controlling the sensitivity of the metric (greater $m$ reduces sensitivity). We recommend the value of $m$ to be in the range between 2 and 3. The projection 
presented in Fig. 3c was detected with this method damages present in phase are also visible in the amplitude. This metric is particularly effective in detecting projections which do not contain much of high phase gradients and have approximately constant amplitude distribution with respect to illumination direction.

After sifting the dataset with the above described metrics, we sort the erroneous projections within each metric and delete all of them or until the specified limit is reached (limit can be set by user, up to $30 \%$ number of projections by default), as removing too many projections also negatively impacts the reconstruction, especially if it leads to uneven filling of the spectral domain [7].
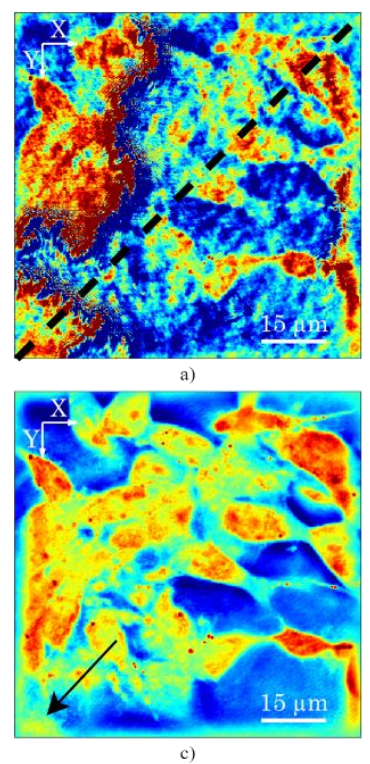

Fig. 4. Reconstructions: a) original, b) after using spectrum metric only, c) after using all metrics, d) refractive index along diagonal marked on panel a).

The described algorithm allowed for significant quality improvement of reconstruction of measurements, which contained very erroneous projections. The removal of these projections is crucial, as we can see in Figs. 4a and $4 \mathrm{~b}$. In the case of slightly damaged projections, it is not obvious which projections should yet be removed and which not, as we must consider the loss of information contained by the non-damaged part of a projection. In the case presented in Fig. 4c, we had errors accumulated in the lower left corner of the reconstruction, due to the phase unwrapping errors in around a dozen of projections, as seen in Fig. 2c. The removal of these slightly damaged projections did not improve the quality of reconstruction and resulted in noticeable local changes. Overall impact on the reconstruction, of projections similar to the one presented in Fig. 2c, is highly dependent on the amount of damages, their severity, as well as location. If damages appear repeatedly in certain region of projections, then their effect accumulates in reconstruction, but if damages are not severe and scattered in projections, their effect on reconstruction can be negligible. In the ideal case, a set of projections which ensure the best possible quality of reconstruction should be passed to the reconstruction algorithm. As currently there is no objective and definitive method for assessing where is the boundary delimiting projections which yet should be removed from the ones which should not, we included a few users controlled parameters in the algorithm. Those parameters control the sensitivity of each metric giving the user a possibility of adjustments as needed. To give an example, one can have lower screening sensitivity when dealing with smaller datasets where the reconstruction quality is limited by the measurement time and noise level, or higher sensitivity when more projections are available and the objective is to achieve the best possible quality.

Overall, the designed algorithm significantly improves the quality of reconstruction whenever badly erroneous projections are present in the dataset making the most of the originally acquired data.

The described methods are useful not only for the screening of holographic projections captured in LAHT systems, but they can be also implemented for other techniques that rely on capturing large datasets of similar complex fields, such as long time-lapse monitoring at digital holographic microscopes or cameras.

This work has been supported by TEAM TECH/20161/4 of Foundation for Polish Science, co-financed by the European Union under the European Regional Development Fund.

\section{References}

[1] A. Kuś, W. Krauze, P.L. Makowski, M. Kujawińska, ETRI J. 41, 1 (2019).

[2] V. Balasubramani et al., Appl. Opt. 60, 10 (2021).

[3] Y. Park, C. Depeursinge, G. Popescu, Nature Photonics 12, 10 (2018).

[4] W. Krauze, P. Makowski, M. Kujawińska, A. Kuś, Opt. Expr. 24, 5 (2016).

[5] D. Ryu et al., Sci Rep. 9, 1 (2019).

[6] B.S. Lipkin, Picture Processing and Psychopictorics. (Saint Louis, Elsevier Science 2014).

[7] A.M. Taddese, N. Verrier, M. Debailleul, J.-B. Courbot, O. Haeberlé, Appl. Opt. 60, 6 (2021). 